\title{
Effects of desynchronized sleep deprivation upon startle response habituation in the rat*
}

\author{
WARREN C. STERN† \\ Indiana University, Bloomington, Ind. 47401
}

Rats deprived of desynchronized (D) sleep had faster habituation rates of click-induced startle responses than did normals, but had the same habituation rates as cold water immersed stress controls. Faster habituation in the D sleep deprived group is probably not due to $D$ sleep loss per se, but rather to the accompanying stress and/or increased activity levels.

Sleep in the rat consists of at least two distinct states. Synchronized sleep $(\mathrm{S})$ is characterized, in part, by a synchronous neocortical and hippocampal electroencephalogram (EEG) and slow eye movements. Desynchronized sleep (D, REM sleep), on the other hand, is accompanied by a desynchronized neocortical EEG, theta rhythm in the hippocampus, and rapid eye movements. While there have been many investigations of the physiological correlates of $\mathbf{S}$ and $D$ sleep, comparatively little is known about the significance of these states for subsequent behavior while awake. The present report is one of a series of studies examining the behavioral effects of $D$ deprivation in the rat (Stern, 1970a, b). Startle response habituation to repeated clicks were measured following 5 days of $D$ deprivation, stress control, and normal treatments.

It was hypothesized that, following $D$ deprivation, rats would require more clicks to produce habituation of the startle response than would normals because: (1) habituation is considered to be a form of learning (Sokolov, 1963; Thompson \& Spencer, 1966) and $D$ deprivation can produce learning impairments in rats (Stern, $1970 \mathrm{~b})$; (2) startle responses are a measure of emotional reactivity, and after $D$ deprivation rats show increased emotionality (Morden, Conner, Mitchell, Dement, \& Levine, 1968; Albert, Siegel, \& Cicala, 1970; Stern, 1970a); (3) following D deprivation, the auditory system may show increased excitability, since D-deprived cats have faster auditory cortex recovery cycles of click-evoked potentials (Dewson, Dement, Wagener, \& Nobel, 1967).

*This research was supported in part by NIMH Grant MH 14658 to Indiana University and by NB 03097-05 to the Worcester Foundation for Experimental Biology. The author wishes to thank Dr, G. P. Frommer for his advice.

tRequests for reprints should be addressed to Warren $C$. Stern, Worcester Foundation for Experimental Biology, Shrewsbury, Mass. 01545 .

\section{SUBJECTS}

Fifty-six naive male Sprague-Dawley rats, supplied by Hormone Assay at an age of 65-90 days, were employed.

APPARATUS AND PROCEDURE Habituation Testing

Startle responses were electrically transduced by a phonograph cartridge attached to an (activity) platform mounted on synthetic sponge. Electrical signals resulting from displacements of the activity platform by rat movements were recorded on a Grass Model 7 polygraph, with a high-frequency cutoff of $3 \mathrm{~Hz}$ and a paper speed of $25 \mathrm{~mm} / \mathrm{min}$. The occurrence of a startle response (a pen deflection at least $1 \mathrm{~mm}$ above background activity level) was scored by an $O$, who had knowledge of S's treatment, as the clicks were delivered. The recording system produced a 9-mm peak-to-peak pen deflection in response to a $3.1-\mathrm{g}$ weight dropped $10 \mathrm{~cm}$ onto the platform. Rats were placed individually in a $21 \times 21 \times 28 \mathrm{~cm}$ wire cage, located on the activity platform. The cage and platform were enclosed in an illuminated sound-attenuating refrigerator shell. The closest edge of the rat's cage was $30 \mathrm{~cm}$ from a speaker which presented 0.5 -msec pulsed clicks. The clicks were produced by Tektronix waveform generators and amplifiers, with the peak-to-peak amplitude of the click equal to the peak-to-peak amplitude of 95-dB white noise.

Habituation testing was preceded by a 5-min adaptation period on the activity platform. Following adaptation, clicks were presented at 10-sec intervals, until the occurrence of no startle response on two consecutive clicks (the habituation criterion) or until 100 clicks occurred. Treatments

D deprivation was produced by confining rats for 5 days to a 5- to 6-cm-diam platform located $1 / 2-2 \mathrm{~cm}$ above the water level in an escape-proof enclosure. The effectiveness of the present "water tank" deprivation technique has been demonstrated by Jouvet, Vimont,
Delorme, \& Jouvet (1964) and Morden, Mitchell, \& Dement (1967). Food was continuously available from an overhead wire mesh screen. A sleep recovery group was given 4 days of sleep recovery following 5 days of $D$ deprivation prior to startle-response habituation testing. Normal rats were confined to their home cages for 5 days with ad lib food and water. A stress control group was immersed in cold water for $1 \mathrm{~h}$ per day for 5 days (mean temperature $=19^{\circ} \mathrm{C}$, depth of $10 \mathrm{~cm}$ ) with the remaining time spent in the home cages. This treatment produced an adrenal gland hypertrophy, and restricted food intake produced a body weight loss which was approximately equal to that of the 5-day D-deprived rats (Stern, 1970a).

Polygraphic recordings of the cortical and hippocampal EEGs and activity levels from two Ss showed that D sleep changed relatively little (less than 25\% decrease) during the 5-day stress treatment. Another group was confined to an 11.5-cm-diam platform, located at water level in the water tank apparatus employed to produce $D$ deprivation. This traditionally employed large-platform stress control, which had an adrenal hypertrophy and body weight loss similar to that of the 5 -day $\mathrm{D}$-deprived group (Stern, 1970a), experiences large losses of D sleep (Duncan, Henry, Karadzic, Mitchell, Pivik, Cohen, \& Dement, 1967) and is, therefore, an inadequate stress control (see Stern, $1970 \mathrm{~b})$. The large-platform condition was included in the present experiment for heuristic purposes.

Habituation testing began between 15 and $60 \mathrm{~min}$ following removal from the water tank apparatus for the D-deprived and large-platform groups and 2-4 $\mathrm{h}$ after the end of the last cold water immersion in the stress control group.

\section{RESULTS}

The number of clicks delivered prior to the start of the habituation criterion (two consecutive clicks which failed to elicit a startle response) are presented in Table 1, Part A. Habituation of the normal and sleep recovery groups required an average of 29 and 26 clicks, respectively, while the D-deprived, large-platform, and cold-water-stress Ss received an average of only 9,2 , and 3 clicks, respectively. A chi-square analysis of the number of Ss per group with habituation scores greater than or equal to (vs less than) the overall mean of the habituation scores $(14.8$ clicks $)$ indicated significant treatment differences $\left(x^{2}=23.29, d f=4, p<.001\right)$. Three chi-square comparisons (using 2 by 2 contingency tables, Siegel, 1956) of the number of clicks to habituation in 
Table 1

Startle Response Habituation and Activity Levels

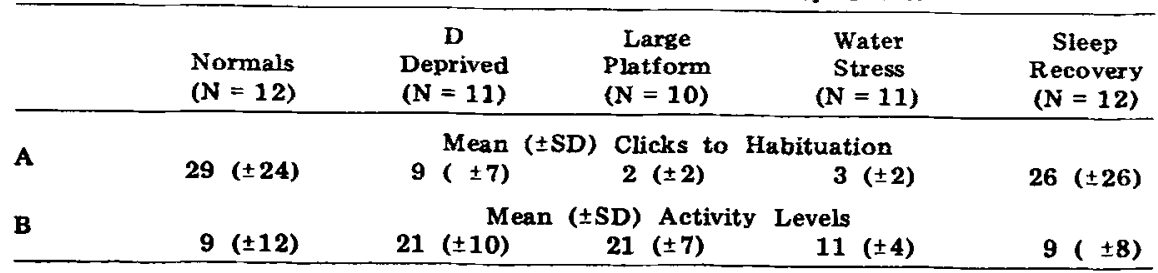

the D-deprived group to those of the three other treatments showed that only normals received significantly more clicks than the $D$-deprived rats $\left(x^{2}=6.03, \mathrm{df}=1, \mathrm{p}<.02\right)$. However, the sleep recovery group showed a strong trend towards slower habituation rates than the $D$-deprived group $\left(x^{2}=3.19, \mathrm{df}=1, \mathrm{p}<.10\right)$.

General activity scores were obtained during the interclick intervals of the first 20 clicks. An activity index, consisting of the sum of five randomly chosen measurements per $S$ (with the restriction that the measurements be made during the interval from 2 sec after a click to 2 sec before the start of the next click) is presented in Table 1 , Part $B$. The activity level of the $\mathrm{D}$-deprived and large-platform rats was approximately twice that of the normal, cold-water-stress, and sleep recovery groups. A Kruskal-Wallis analysis of variance of these activity scores showed significant differences $(\mathrm{H}=21.3, \quad \mathrm{df}=4, \quad \mathrm{p}<.001)$. Chi-square tests ( 2 by 2 contingency tables) showed that normals had significantly less activity than both D-deprived $\left(x^{2}=8.3, \mathrm{df}=1, \mathrm{p}<.01\right)$ and large platform $S s\left(x^{2}=8.3, d f=1\right.$, $\mathrm{p}<.01$ ).

Parts $A$ and $B$ of Table 1 show an inverse relationship between the number of clicks to habituation and the interclick-interval activity levels. The groups which required the fewest clicks before habituation tended to have the highest activity, and vice versa. A Spearman rank-order correlation between the number of clicks to habituation and the interclick-interval activity scores of all 56 rats was -.46 (correction for ties employed), which was significant $(\mathrm{t}=3.4, \mathrm{df}=54, \mathrm{p}<.001)$.

$$
\text { DISCUSSION }
$$

The startle response habituation rates of the D-deprived, large-platform confined, and cold-water-immersed stress control groups were greater than those of normal rats. The results of the D-deprived group agree with the finding that rats deprived of both $D$ and $S$ sleep (partial sleep deprivation) for up to 28 days emitted fewer startle responses to loud sounds (Webb, 1962). However, the present effects of the D-deprivation and large-platform confinement treatments may be due to the following: (1) Stress-the three groups that showed faster habituation (the D-deprived, large-platform, and cold-water-stress controls) showed similar weight loss and adrenal hypertrophy. (2) An increase in activity levels in the D-deprived and large-platform groups. Their greater activity, perhaps due to increased grooming, may have produced movements which masked the occurrence of startle responses or induced postures which were incompatible with the performance of a startle response. Additional support for the activity-level interpretation is that a negative correlation was found between the number of clicks required to produce habituation and the activity levels of the Ss. Rats that were highly active (and were most frequently found in the D-deprived and large-platform groups) tended to require the fewest clicks for habituation. (3) Faster learning in the D-deprived groups: this is unlikely since previous studies of acquisition performance following $D$ deprivation, using tasks less sensitive to disruption by changes in activity levels or emotionality, report either slower acquisition or no effect (see Stern, $1970 \mathrm{~b}$ ). (4) Decreased emotionality in the D-deprived groups: this is unlikely since $D$-deprived rats show increased emotionality, based upon findings of hyperreactivity to handling, increased aggressiveness (Morden et al, 1968; Stern, 1970a), and increased reactivity to electric shock (Albert et al, 1970; Stern, 1970a). In summary, while the $D$-deprivation treatment produced faster habituation, this effect was probably not due to $D$ sleep loss per se, but rather to the accompanying stress and weight loss or to increased activity levels.

The activity and habituation performance of the large-platform confined Ss were remarkably similar to that of the small-platform D-deprived group. This is probably due to the similar amounts of $\mathrm{D}$ deprivation, stress, and weight loss which occurred in these two groups. Other studies, using different behavioral tests, have also reported that the large-platform and $D$-deprived groups tend to show similar behavioral changes (Albert et al, 1970; Stern, 1970a).

\section{REFERENCES}

ALBERT, I., CICALA, G. A., \& SIEGEL, J. The behavioral effects of REM sleep deprivation in rats. Psychophysiology, $1970,6,550-560$.

DEWSON, J. H., III, DEMENT, W. C. WAGENER, T., \& NOBEL, K. Rapid eye movement sleep deprivation: A central-neural change during wakefulness. Science, 1967, 156, 403-406.

DUNCAN, R., HENRY, P., KARADZIC, V. MITCHELL, G., PIVIK, T., COHEN, H. \& DEMENT, W. Baseline sleep and REM deprivation in the rat. Paper presented at the meeting of the Association for the Psychophysiological Study of Sleep, Santa Monica, California, March 1967.

JOUVET, D., VIMONT, P., DELORME, F. \& JOÚVET, M. Etude de la privation selective de la phase paradoxale de sommeil chez le chat. Comptes Rendus del la Société de Biologie, 1964, 158 . 756-759.

MORDEN, B., CONNER, R., MITCHELL, G., DEMENT, W., \& LEVINE, S. Effects of rapid eye movement (REM) sleep deprivation on shock-induced fighting Physiology \& Behavior, 1968, 3, 425.432.

MORDEN, B., MITCHELL, G., \& DEMENT, W Selective REM sleep deprivation and compensation phenomena in the rat. Brain Research, $1967,5,339-349$.

SIEGEL, S. Nonparametric stat istics for the behovioral sciences. New York: MeGraw-Hill, 1956.

SOKOLOV, Y. N. Perception and the conditioned reflex. New York: Macmillan, 1963.

STERN, W. C. Behavioral and biochemical aspects of rapid eye movement sleep deprivation in the rat. Doctoral dissertation, Indiana University. Ann Arbor, Michigan: University Microfilms, 1970a. No. 70-14971.

STERN, W. C. The relationship between REM sleep and learning: Animal studies. In E. Hartmann (Ed.). Sleep and dreaming, Boston: Little Brown, 1970b.

THOMPSON, R. F., \& SPENCER, W. A. Habituation: A model phenomenon for the study of of neuronal substrates of behavior. Psychological Review, 1966, 173, 16-43.

WEBB, W. B. Some effects of prolonged sleep deprivation on the hooded rat. Journal of Comparative \& Physiological Psychology, 1962, 55, 791-793. 